\title{
LARGE TIME BEHAVIOR AND GLOBAL EXISTENCE OF SOLUTION TO THE BIPOLAR DEFOCUSING NONLINEAR SCHRÖDINGER-POISSON SYSTEM
}

\author{
$\mathrm{BY}$
}

CHENGCHUN HAO AND LING HSIAO

Academy of Mathematics and Systems Science, CAS, Beijing, 100080, P. R. China

\begin{abstract}
In this paper, we study the large time behavior and the existence of globally defined smooth solutions to the Cauchy problem for the bipolar defocusing nonlinear Schrödinger-Poisson system in the space $\mathbb{R}^{3}$.
\end{abstract}

1. Introduction. In the present paper, we study the global existence and large time behavior for the bipolar defocusing nonlinear Schrödinger-Poisson (BDNLSP) system

$$
\begin{aligned}
& i \varepsilon \dot{\psi}_{j}=-\frac{\varepsilon^{2}}{2} \Delta \psi_{j}+\left(q_{j} V+h_{j}\left(\left|\psi_{j}\right|^{2}\right)\right) \psi_{j}, \quad j=1,2, \\
& -\lambda^{2} \Delta V=\left|\psi_{1}\right|^{2}-\left|\psi_{2}\right|^{2},
\end{aligned}
$$

with the initial data

$$
\psi_{j}(0, \cdot)=\varphi_{j}, \quad j=1,2,
$$

where the wave function $\psi_{j}=\psi_{j}(t . x): \mathbb{R}^{1+d} \rightarrow \mathbb{C}, j=1,2, \dot{\psi}_{j}=\partial \psi_{j} / \partial t, \Delta$ is the Laplace operator on $\mathbb{R}^{d}$, and the electrostatic potential $V=V(t, x)$. The nonlinear self-interacting potential $h_{j}(s)$ is assumed to be given by

$$
h_{j}(s)=a_{j}^{2} s^{\gamma, j}, \quad \text { for } s \geqslant 0 \text { and some } a_{j}>0, \frac{2}{d}<\gamma_{j}<\alpha(d),
$$

where $\alpha(d)=\frac{2}{d-2}$ if $d \geqslant 3$ and $\alpha(d)=\infty$ if $d=1,2$. The charges of the particles described by the wave functions $\psi_{j}$ are defined by $q_{1}=1, q_{2}=-1$, respectively. $\varepsilon$ is the scaled Planck constant and $\lambda$ is the scaled Debye length.

Received July 31, 2003.

2000 Mathematics Subject Classification. Primary 35Q55, 82D10.

Key words and phrases. Bipolar defocusing nonlinear Schrödinger-Poisson system, global existence, large time behavior, pseudo-conformal conservation law, the Galilei-type operator.

L. Hsiao is supported by the MST (Grant No. 1999075107), NSAF (Grant No. 10276036), and the Innovation funds of AMSS, CAS of China.

E-mail address: hcc@mail.amss.ac.cn

E-mail address: hsiaol@mail.amss.ac.cn 
We assume that the initial value

$$
\varphi_{j}(x) \in \Sigma\left(\mathbb{R}^{d}\right):=\left\{u \in H^{1}\left(\mathbb{R}^{d}\right):|x| u \in L^{2}\left(\mathbb{R}^{d}\right)\right\}, j=1,2,
$$

with the norm

$$
\left\|\psi_{j}\right\|_{\Sigma}=\left\|\psi_{j}\right\|_{H^{1}}+\left\||x| \psi_{j}\right\|_{L^{2}} .
$$

This system appears in quantum mechanics as well as semi-conductor and plasma physics. A large amount of interesting work has been devoted to the study of the Schrödinger-Poisson systems (see [2], [3], [4], [6], [7] and references therein). In [4], by applying the estimates of a modulated energy functional and the Wigner measure method, Jüngel and Wang discussed the combined semi-classical and quasineutral limit of the (BDNLSP) system with the initial data (1.3) in the whole space where $a_{1}=a_{2}$ and $\gamma_{1}=\gamma_{2}$, provided the solution of (1.1)-(1.3) exists. But they only declared the existence and uniqueness of global small smooth solution under the assumption that the initial data were sufficiently small in $H^{s}$ where $s>d / 2+2$. And in [3], Castella proved the global existence and the asymptotic behavior of solutions in the function space $L^{2}$ for the mixed-state unipolar Schrödinger-Poisson systems without the defocusing nonlincarity. In [6], with the help of madelung transform and WKB expansion, Li and Lin discussed the following unipolar nonlinear Schrödinger-Poisson system:

$$
\begin{aligned}
& i \varepsilon \psi_{t}^{\varepsilon}+\frac{\varepsilon^{2}}{2} \Delta \psi^{\varepsilon}-\left(V^{\varepsilon}(x, t)+f^{\prime}\left(\left|\psi^{\varepsilon}\right|^{2}\right)\right) \psi^{\varepsilon}-\left(\arg \psi^{\varepsilon}\right) \psi^{\varepsilon}=0, \\
& -\Delta V^{\varepsilon}=\left|\psi^{\varepsilon}\right|^{2}-\mathcal{C}(x), \quad V \rightarrow 0 \text { as }|x| \rightarrow \infty,
\end{aligned}
$$

subject to the rapidly oscillating (WKB) initial condition

$$
\psi^{\varepsilon}(x, 0)=\psi_{0}^{\varepsilon}=A_{0}^{\varepsilon}(x) e^{\frac{i}{\varepsilon} S_{0}(x)},
$$

where $f \in \mathcal{C}^{\infty}\left(\mathbb{R}^{+} ; \mathbb{R}\right), S_{0} \in H^{s}\left(\mathbb{R}^{d}\right), d \geqslant 1$. for $s \geqslant d / 2+2, A_{0}^{\varepsilon}$ was a function, polynomial in $\varepsilon$, with coefficients of Sobolev regularity in $x$, and the function $C(x)>0$ denoted the background ions. They obtained the existence of smooth solution where the wave function was of the form $\psi^{\varepsilon}(x, t)=A^{\varepsilon}(x, t) e^{\frac{i}{\varepsilon} S^{\varepsilon}(x, t)}$, with $A^{\varepsilon}$ and $\nabla S^{\varepsilon}$ bounded in $L^{\infty}\left([0 . T] ; H^{s}\left(\mathbb{R}^{d}\right)\right)$ and the initial data being sufficiently small in $H^{s}\left(\mathbb{R}^{d}\right)$. However, to our knowledge, there is no previous result on the global existence and the asymptotic behavior of solutions for the (BDNLSP) system with arbitrary initial data in $\Sigma\left(\mathbb{R}^{3}\right)$. In this paper, by using the pseudo-conformal conservation law of the (BDNLSP) system and applying the time-space $L^{p}-L^{p^{\prime}}$ estimate method, we shall establish the global existence and uniqueness of the solution to the (BDNLSP) system with initial data in $\Sigma\left(\mathbb{R}^{3}\right)$. As a byproduct, the large time behavior to the solution is also obtained. Although the above results are established for the single bipolar defocusing nonlinear Schrödinger-Poisson system, the results can be extended to the mixed-state bipolar defocusing nonlinear Schrödinger-Poisson system within the same framework.

For convenience, we first introduce some notation. For any $p \in[2, \infty)$, we denote $\frac{1}{\gamma(p)}=\frac{3}{2}\left(\frac{1}{2}-\frac{1}{p}\right)$. $S(t)$ denotes the unitary group generated by $\frac{\varepsilon}{2} i \Delta$ in $L^{2}\left(\mathbb{R}^{3}\right)$. For $p \in[1, \infty]$, we denote by $p^{\prime}$ the conjugate exponent of $p$, defined by $1 / p+1 / p^{\prime}=1 . \bar{z}$ denotes the conjugate of the complex number $z$. 
Now we state the main result of this paper.

Theoren 1.1 (Existence and uniqueness). Let $\varphi_{j} \in \Sigma\left(\mathbb{R}^{3}\right)$. Assume that $\rho \in[2,6)$. Then, there exists a unique solution

$$
\psi_{j} \in \mathcal{C}\left(\mathbb{R}: \Sigma\left(\mathbb{R}^{3}\right)\right) \cap L^{\infty}\left(\mathbb{R} ; H^{1}\left(\mathbb{R}^{3}\right)\right) \cap L_{l o c}^{\gamma(\rho)}\left(\mathbb{R} ; H_{\rho}^{1}\left(\mathbb{R}^{3}\right)\right) \text {, for } j=1.2
$$

to the (BDNLSP) system with the initial data (1.3).

Moreover, the solution $\left(\psi_{1}, \psi_{2}, V\right)$ satisfies the $L^{2}$-norm, the cnergy, and the pseudoconformal conservation laws (for details, one can see Proposition 2.1 in the case $d=3$ ).

TheOrem 1.2 (Large time behavior). Let $\left(\psi_{1}, \psi_{2}, V\right)$ and $\rho$ be as in Theorem 1.1. Then, there exist constants $C$ depending only on $\left\|\varphi_{j}\right\|_{H^{1}}$ and $\left\||x| \varphi_{j}\right\|_{2}$ such that

$$
\begin{aligned}
& \left\|\psi_{j}\right\|_{\rho} \leqslant C|t|^{-\frac{1}{\gamma(\rho)}}, \quad \forall \rho \in[2,6), \forall|t| \geqslant 1, \\
& \|\nabla V(t)\|_{\rho} \leqslant C|t|^{-\left(1-\frac{3}{2 \rho}\right)}, \quad \forall \rho \in\left(\frac{3}{2}, \infty\right), \forall|t| \geqslant 1, \\
& \|V(t)\|_{\rho} \leqslant C|t|^{-\frac{1}{2}\left(1-\frac{3}{\rho}\right)}, \quad \forall \rho \in(3, \infty), \forall|t| \geqslant 1 .
\end{aligned}
$$

In the next section we shall derive three conservation identities including the $L^{2}$-norm, the energy, and the pseudo-conformal conservation laws in the whole space $\mathbb{R}^{d}$ for any $d \in \mathbb{N}$. In Sec. 3, we will give some basic estimates used in our proofs for $d=3$. Section 4 is devoted to the proof of the existence and uniqueness of the solutions for the initial data in $\Sigma\left(\mathbb{R}^{3}\right)$. Finally, the large time behavior of the solution is obtained in Sec. 5 .

\section{Derivation of the conservation laws.}

Proposition 2.1. Let $d \in \mathbb{N},\left\{\psi_{j}\right\}$ be a solution of the (BDNLSP) system with the initial value $\varphi_{j}(x) \in \Sigma\left(\mathbb{R}^{d}\right)$. Then, we have the following conservation laws for all $t \in \mathbb{R}$ :

(i) $L^{2}$-norm law:

$$
\left\|\psi_{j}(t)\right\|_{2}=\left\|\varphi_{j}\right\|_{2} \quad \text { for } j=1,2
$$

(ii) Energy conservation law:

$$
\varepsilon^{2} \sum_{j=1}^{2}\left\|\nabla \psi_{j}(t)\right\|_{2}^{2}+\lambda^{2}\|\nabla V\|_{2}^{2}+2 \sum_{j=1}^{2} \frac{a_{j}^{2}}{\gamma_{j}+1}\left\|\psi_{j}(t)\right\|_{2\left(\gamma_{j}+1\right)}^{2\left(\gamma_{j}+1\right)}=\text { const; }
$$

(iii) Pseudo-conformal conservation law (cf. [9]):

$$
\begin{aligned}
\sum_{j=1}^{2}\left\|x \psi_{j}+i \varepsilon t \nabla \psi_{j}\right\|_{2}^{2} & +\lambda^{2} t^{2}\|\nabla V\|_{2}^{2}+2 t^{2} \sum_{j=1}^{2} \frac{a_{j}^{2}}{\gamma_{j}+1}\left\|\psi_{j}\right\|_{2\left(\gamma_{j}+1\right)}^{2\left(\gamma_{j}+1\right)} \\
& +2 \sum_{j=1}^{2} \frac{a_{j}^{2}\left(d \gamma_{j}-2\right)}{\gamma_{j}+1} \int_{0}^{t} \tau\left\|\psi_{j}(\tau)\right\|_{2\left(\gamma_{j}+1\right)}^{2\left(\gamma_{j}+1\right)} \mathrm{d} \tau \\
= & \sum_{j=1}^{2}\left\||x| \varphi_{j}\right\|_{2}^{2}+(4-d) \lambda^{2} \int_{0}^{t} \tau\|\nabla V(\tau)\|_{2}^{2} \mathrm{~d} \tau .
\end{aligned}
$$


Proof. Denote eq $\left(\psi_{j}\right)=: i \varepsilon \dot{\psi}_{j}+\frac{\xi^{2}}{2} \Delta \psi_{j}-\left(q_{j} V+h_{j}\left(\left|\psi_{j}\right|^{2}\right)\right) \psi_{j}$.

(i) It is well known that (2.1) holds for $j=1,2$. We omit its proof.

(ii) We consider

$$
\Re\left(c^{\prime} q\left(l_{j}\right) \cdot \dot{\psi}_{j}\right)=0
$$

where $(\cdot, \cdot)$ denotes the $L^{2}$-imner product. From the above, we can get

$$
\int_{\mathbb{R}^{d}}\left\{\frac{\varepsilon}{4} \partial_{t}\left|\nabla \iota_{j}\right|^{2}+\frac{1}{2}\left(q_{j} V+h_{j}\left(\left|\psi_{j}\right|^{2}\right)\right) \partial_{t}\left|\psi_{j}\right|^{2}\right\} \mathrm{d} x=0 .
$$

Hence,

$$
\begin{aligned}
\int_{\mathbb{R}^{d}}\left\{\frac { \varepsilon } { 4 } \partial _ { t } \left(\left|\nabla \psi_{1}\right|^{2}\right.\right. & \left.+\left|\nabla \psi_{2}\right|^{2}\right)+\frac{1}{2} V \partial_{t}\left(\left|\psi_{1}\right|^{2}-\left|\psi_{2}\right|^{2}\right) \\
& \left.+\frac{1}{2} h_{1}\left(\left|\psi_{1}\right|^{2}\right) \partial_{t}\left|\psi_{1}\right|^{2}+\frac{1}{2} h_{2}\left(\left|\psi_{2}\right|^{2}\right) \partial_{t}\left|\psi_{2}\right|^{2}\right\} \mathrm{d} x=0 .
\end{aligned}
$$

Integrating (2.4) over $[0, t]$, we obtain the desired identity.

(iii) Considering

$$
\Re\left(e q\left(\psi_{j}\right) \bar{\psi}_{j}\right)=0
$$

we have

$$
-\varepsilon \Im \partial_{t} \psi_{j} \bar{\psi}_{j}+\frac{\varepsilon^{2}}{2} \Re \nabla \cdot\left(\nabla \psi_{j} \bar{\psi}_{j}\right)-\frac{\varepsilon^{2}}{2}\left|\nabla \psi_{j}\right|^{2}-\left(q_{j} V+h_{j}\left(\left|\psi_{j}\right|^{2}\right)\right)\left|\psi_{j}\right|^{2}=0 .
$$

Noticing

$$
\Re\left(e q\left(\psi_{j}\right) \bar{\psi}_{j}, r\right)=0 \quad \text { with } r:=|x|
$$

we obtain

$$
\begin{aligned}
-\varepsilon S \partial_{t} \psi_{j} \bar{\psi}_{j r} r+\frac{\varepsilon^{2}}{2} \Re \nabla \cdot\left(\nabla \psi_{j} \bar{\psi}_{j} r\right. & \left.-\frac{x}{2}\left|\nabla \psi_{j}\right|^{2}\right)+\frac{\varepsilon^{2}}{4}(d-2)\left|\nabla \psi_{j}\right|^{2} \\
& -\frac{1}{2}\left(q_{j} V+h_{j}\left(\left|\psi_{j}\right|^{2}\right)\right) r \partial_{r}\left|\psi_{j}\right|^{2}=0 .
\end{aligned}
$$

Due to

$$
\partial_{t}\left(\psi_{j} \bar{\psi}_{j} r r\right)+\nabla \cdot\left(x \bar{\psi}_{j} \partial_{t} \psi_{j}\right)=2 \partial_{t} \psi_{j} \bar{\psi}_{j}{ }_{j} r+2 \Re \psi_{j} x \cdot \nabla \partial_{t} \bar{\psi}_{j}+d \partial_{t} \psi_{j} \bar{\psi}_{j},
$$

we have, by taking the imaginary part, that

$$
\Im \partial_{t}\left(\psi_{j} \bar{\psi}_{j r} r\right)+\Im \nabla \cdot\left(x \bar{\psi}_{j} \partial_{t} \psi_{j}\right)=d \Im \partial_{t} \psi_{j} \bar{\psi}_{j}+2 \Im\left(\partial_{t} \psi_{j} \bar{\psi}_{j r} r\right) .
$$

From (2.5) and (2.6), we have

$$
\begin{aligned}
-\varepsilon \Im \partial_{t}\left(\psi_{j} \bar{\psi}_{j r} r\right) & -\Im \nabla \cdot\left(x \bar{\psi}_{j} \partial_{t} \psi_{j}\right)-\frac{\varepsilon^{2}}{2} d \Re \nabla \cdot\left(\nabla \psi_{j} \bar{\psi}_{j}\right)-d\left(q_{j} V+h_{j}\left(\left|\psi_{j}\right|^{2}\right)\right)\left|\psi_{j}\right|^{2} \\
& +\varepsilon^{2} \Re \nabla \cdot\left(\nabla \psi_{j} \bar{\psi}_{j r} r-\frac{x}{2}\left|\nabla \psi_{j}\right|^{2}\right)-\left(q_{j} V+h_{j}\left(\left|\psi_{j}\right|^{2}\right)\right) r \partial_{r}\left|\psi_{j}\right|^{2}=0 .
\end{aligned}
$$

We also have

$$
\partial_{t}\left|x \psi_{j}+i \varepsilon t \nabla \psi_{j}\right|^{2}=\partial_{t}\left(\left|\psi_{j} r\right|^{2}+\varepsilon^{2} t^{2}\left|\nabla \psi_{j}\right|^{2}+2 \varepsilon t \Im \psi_{j} x \cdot \nabla \bar{\psi}_{j}\right) .
$$


Since

$$
\begin{aligned}
\frac{\varepsilon}{2} \partial_{t}\left|\psi_{j} r\right|^{2} & =\varepsilon \Re\left(\partial_{t} \psi_{j} \bar{\psi}_{j}\right) r^{2}=\Im i \varepsilon \partial_{t} \psi_{j} \bar{\psi}_{j} r^{2} \\
& =\Im\left[-\frac{\varepsilon^{2}}{2} \Delta \psi_{j}+\left(q_{j} V+h_{j}\left(\left|\psi_{j}\right|^{2}\right)\right) \psi_{j}\right] \bar{\psi}_{j} r^{2} \\
& =-\frac{\varepsilon^{2}}{2} \Im \Delta \psi_{j} \bar{\psi}_{j} r^{2}, \\
\Im \nabla \cdot\left(\nabla \psi_{j} \bar{\psi}_{j} r^{2}\right) & =\Im \Delta \psi_{j} \bar{\psi}_{j} r^{2}-2 \Im\left(\nabla \bar{\psi}_{j} \cdot x\right) \psi_{j},
\end{aligned}
$$

we have

$$
\begin{aligned}
\partial_{t}\left|x \psi_{j}+i \varepsilon t \nabla \psi_{j}\right|^{2}=2 \varepsilon^{2} t\left|\nabla \psi_{j}\right|^{2} & +\varepsilon^{2} t^{2} \partial_{t}\left|\nabla \psi_{j}\right|^{2}-\varepsilon \Im \nabla \cdot\left(\nabla \psi_{j} \bar{\psi}_{j} r^{2}\right) \\
& +2 \varepsilon t \Im \partial_{t}\left(\psi_{j} x \cdot \nabla \bar{\psi}_{j}\right) .
\end{aligned}
$$

Integrating (2.7) over $\mathbb{R}^{d}$, we obtain that for $j=1,2$

$$
\begin{aligned}
\partial_{t}\left\|x \psi_{j}+i \varepsilon t \nabla \psi_{j}\right\|_{2}^{2}=\varepsilon^{2} t^{2} \partial_{t}\left\|\nabla \psi_{j}\right\|_{2}^{2}-2 t \int_{\mathbb{R}^{d}}\{ & d\left(q_{j} V+h_{j}\left(\left|\psi_{j}\right|^{2}\right)\right)\left|\psi_{j}\right|^{2} \\
& \left.+\left(q_{j} V+h_{j}\left(\left|\psi_{j}\right|^{2}\right)\right) r \partial_{r}\left|\psi_{j}\right|^{2}\right\} \mathrm{d} x .
\end{aligned}
$$

From the above, we obtain

$$
\begin{aligned}
\partial_{t} \sum_{j=1}^{2} \| x \psi_{j} & +i \varepsilon t \nabla \psi_{j} \|_{2}^{2}+4 t^{2} \partial_{t}\left(\frac{\lambda^{2}}{4}\|\nabla V\|_{2}^{2}+\sum_{j=1}^{2} \frac{a_{j}^{2}}{2\left(\gamma_{j}+1\right)}\left\|\psi_{j}\right\|_{2\left(\gamma_{j}+1\right)}^{2\left(\gamma_{j}+1\right)}\right) \\
& +2 t \int_{\mathbb{R}^{d}}\left\{d V\left(-\lambda^{2} \Delta V\right)+V \partial_{r}\left(-\lambda^{2} \Delta V\right) r+d \sum_{j=1}^{2} a_{j}^{2}\left|\psi_{j}\right|^{2\left(\gamma_{j}+1\right)}\right. \\
& \left.+\sum_{j=1}^{2} h_{j}\left(\left|\psi_{j}\right|^{2}\right) r \partial_{r}\left|\psi_{j}\right|^{2}\right\} \mathrm{d} x=0 .
\end{aligned}
$$

Noticing that

$$
h_{j}(s) r \partial_{r} s=a_{j}^{2} s^{\gamma_{j}} r \partial_{r} s=\frac{a_{j}^{2}}{\gamma_{j}+1}\left[\nabla \cdot\left(x s^{\gamma_{j}+1}\right)-d s^{\gamma_{j}+1}\right]
$$

and

$$
\begin{aligned}
\int_{\mathbb{R}^{d}} V r \partial_{r}(-\Delta V) \mathrm{d} x & =\int_{\mathbb{R}^{d}} \nabla V_{r} \cdot \nabla(V r) \mathrm{d} x=\int_{\mathbb{R}^{d}}\left(\nabla V_{r} \cdot \nabla V\right) r+\left(\nabla V_{r} \cdot \nabla r\right) V \mathrm{~d} x \\
& =\int_{\mathbb{R}^{d}} \frac{1}{2} r \partial_{r}|\nabla V|^{2} \mathrm{~d} x+\int_{\mathbb{R}^{d}} V \Delta V \mathrm{~d} x \\
& =\int_{\mathbb{R}^{d}} \frac{1}{2}\left[\nabla \cdot\left(x|\nabla V|^{2}\right)-d|\nabla V|^{2}\right] \mathrm{d} x-\int_{\mathbb{R}^{d}} \nabla V \cdot \nabla V \mathrm{~d} x \\
& =-\left(\frac{d}{2}+1\right)\|\nabla V\|_{2}^{2},
\end{aligned}
$$


we can obtain, in view of $(2.4)$ and $(2.8)-(2.11)$, that

$$
\begin{aligned}
\partial_{t}\left[\sum_{j=1}^{2} \| x \psi_{j}\right. & \left.+i \varepsilon t \nabla \psi_{j}\left\|_{2}^{2}+\lambda^{2} t^{2}\right\| \nabla V\left\|_{2}^{2}+\sum_{j=1}^{2} \frac{2 a_{j}^{2}}{\gamma_{j}+1} t^{2}\right\| \psi_{j} \|_{2\left(\gamma_{j, j}+1\right)}^{2\left(\gamma_{j}+1\right)}\right] \\
& +2 \sum_{j=1}^{2} \frac{a_{j}^{2}\left(d \gamma_{j}-2\right)}{\gamma_{j}+1} t\left\|\psi_{j}(t)\right\|_{2(\gamma, j+1)}^{2\left(\gamma_{j}+1\right)}-(4-l) \lambda^{2} t\|\nabla V(t)\|_{2}^{2}=0 .
\end{aligned}
$$

which yields the pseudo-conformal conservation law.

\section{Basic estimates.}

Lemma 3.1 (Estimate I). Let $\frac{1}{\rho^{\prime}}=\frac{2}{a}+\frac{1}{\rho}+\frac{1}{3}-1, u \cdot v \in L^{\gamma(a)}\left(0, T ; H_{a}^{1}\right)$ and $u \in$ $L^{\gamma(\rho)}\left(0, T: H_{\rho}^{1}\right)$. Then we have the estimate

$$
\begin{aligned}
\left\|\left(\frac{1}{r} * u v\right) w\right\|_{L^{\gamma(p)}\left(0 . T: H_{p^{\prime}}^{1}\right)} \leqslant C T^{1 / 2} & \|u\|_{L^{\gamma^{\prime}(a)}\left(0 . T: H_{a}^{1}\right)} \\
& \|v\|_{L^{\gamma(a)}\left(0 . T: H_{a}^{1}\right)}\left\|w^{\prime}\right\|_{L^{\gamma(p)}\left(0 . T: H_{p}^{1}\right)}
\end{aligned}
$$

Proof. By the known estimate as in [8], we have the following:

$$
\begin{aligned}
\left\|\left(\frac{1}{r} * u v\right) w\right\|_{H_{p^{\prime}}^{1}} & \leqslant C\left\|\frac{1}{r} * u v\right\|_{L^{p}}\|w\|_{H_{q}^{1}}+C\left\|\frac{1}{r} * u v\right\|_{H_{q}^{1}}\|w\|_{L^{p}} \\
& \leqslant C\|u\|_{H_{2 m}^{1}}\|v\|_{H_{2 m}^{1}}\|w\|_{H_{q}^{1}}
\end{aligned}
$$

where $\frac{1}{\rho^{\prime}}=\frac{1}{p}+\frac{1}{q}, \frac{1}{p}=\frac{1}{m}+\frac{1}{3}-1$. Let $2 m=a, q=\rho$. i.e. $\frac{1}{\rho^{\prime}}=\frac{2}{a}+\frac{1}{\rho}+\frac{1}{3}-1$. By the Sobolev cmbedding theorem (cf. [1]), we obtain

$$
\left\|\left(\frac{1}{r} * u v\right) w\right\|_{H_{r^{\prime}}^{1}} \leqslant C\|u\|_{H_{a}^{1}}\|v\|_{H_{a}^{1}}\|w\|_{H_{r}^{1}} .
$$

Since

$$
\frac{1}{\gamma(\rho)^{\prime}}=1-\frac{3}{2}\left(\frac{1}{\rho^{\prime}}-\frac{1}{2}\right)=\frac{1}{2}+\frac{2}{\gamma^{\prime}(\alpha)}+\frac{1}{\gamma(\rho)} .
$$

we have the desired result.

LemMA 3.2 (Estimate II). Let $\rho \in[2.6)$; wo have

$$
\left\||u|^{p} u\right\|_{L^{\gamma(p)^{\prime}\left(0, T: H_{p^{\prime}}{ }^{\prime}\right)}} \leqslant C T^{1-\gamma_{\gamma^{\prime}\left(p^{\prime}\right)}^{2}}\|u\|_{L^{x}\left(0 . T ; H^{1}\right)}^{p}\|u\|_{L^{\gamma(p)}\left(0 . T ; H_{p}^{1}\right)} .
$$

Proof. From the identity

$$
\begin{aligned}
\nabla\left(|u|^{p} u\right) & =\nabla\left(|u|^{p}\right) u+|u|^{p} \nabla u=\frac{p}{2}|u|^{p-2}(\nabla u \bar{u}+u \nabla \bar{u}) u+|u|^{p} \nabla u \\
& =\left(\frac{p}{2}+1\right)|u|^{p} \nabla u+\frac{p}{2}|u|^{p-2} u^{2} \nabla \bar{u} .
\end{aligned}
$$

we have, in view of $\frac{1}{\rho^{\prime}}=\frac{p}{q}+\frac{1}{\rho}$, that

$$
\begin{aligned}
\left\|\nabla\left(|u|^{p} u\right)\right\|_{L^{\rho^{\prime}}} & \leqslant C\left\||u|^{p} \nabla u\right\|_{L^{\rho^{\prime}}}+\left.C\|\| u\right|^{p-2} u^{2} \nabla \bar{u} \|_{L^{p^{\prime}}} \\
& \leqslant C\|u\|_{L^{\prime}}^{p}\|\nabla u\|_{L^{\prime}} .
\end{aligned}
$$

By the Sobolev embedding theorem, it yields

$$
\left\||u|^{p} u\right\|_{H_{r^{\prime}}^{1}} \leqslant C\|u\|_{H^{1}}^{p}\|u\|_{H_{i^{\prime}}^{1}},
$$


which implies the desired result in view of the Hölder inequality with respect to the time variable.

Now we introduce the Galilei-type operator

$$
J(t)=x+i \varepsilon t \nabla .
$$

Let $\mathcal{M}(t)=e^{\frac{i|x|^{2}}{2 \varepsilon t}}$ and $w_{j}=\mathcal{M}(-t) \psi_{j}$; we easily see that

$$
\begin{aligned}
& J(t)=S(t) x S(-t)=\mathcal{M}(t)(i \varepsilon t \nabla) \mathcal{M}(-t), \\
& \left|w_{j}(t)\right|=\left|\psi_{j}(t)\right|, \quad\left|J(t) \psi_{j}(t)\right|=\varepsilon|t|\left|\nabla w_{j}(t)\right| .
\end{aligned}
$$

Lemma 3.3 (Estimate III). It holds

$$
\left\|J\left(\left|\psi_{j}\right|^{p} \psi_{j}\right)\right\|_{L^{\gamma(\rho)^{\prime}}\left(0, T ; L^{\rho^{\prime}}\right)} \leqslant C T^{1-\frac{2}{\gamma(\rho)}}\left\|\psi_{j}\right\|_{L^{\infty}\left(0, T ; H^{1}\right)}^{p}\left\|J \psi_{j}\right\|_{L^{\gamma(\rho)}\left(0, T ; L^{\rho}\right)} .
$$

Proof. We have, in view of (3.5). that

$$
\left\|J\left(\left|\psi_{j}\right|^{p} \psi_{j}\right)\right\|_{L^{\rho^{\prime}}}=\varepsilon|t|\left\|\nabla\left(\left|w_{j}\right|^{p} w_{j}\right)\right\|_{L^{\rho^{\prime}}}
$$

Since

$$
\nabla\left(\left|w_{j}\right|^{p} w_{j}\right)=\nabla\left(\left|w_{j}\right|^{p}\right) w_{j}+\left|w_{j}\right|^{p} \nabla w_{j},
$$

we obtain from Hölder's inequality

$$
\left\|\nabla\left(\left|w_{j}\right|^{p} w_{j}\right)\right\|_{L^{\rho^{\prime}}} \leqslant C\left\|w_{j}\right\|_{H^{1}}^{p}\left\|\nabla w_{j}\right\|_{L^{\rho}} .
$$

Then, by (3.5), (3.7), and (3.8), we see that

$$
\left\|J\left(\left|\psi_{j}\right|^{p} \psi_{j}\right)\right\|_{L^{\rho^{\prime}}} \leqslant C\left\|\psi_{j}\right\|_{H^{1}}^{p}\left\|J \psi_{j}\right\|_{L^{\rho}},
$$

which implies the desired result (3.6) in view of the Hölder inequality with respect to the time variable $t$.

LEMma 3.4 (Estimate IV). We have the estimate

$$
\begin{aligned}
\left\|J\left(V \psi_{j}\right)\right\|_{L^{\gamma(\rho)^{\prime}}\left(0, T ; L^{\rho^{\prime}}\right)} \leqslant C T^{1 / 2}\left\|\left(\psi_{1}, \psi_{2}\right)\right\|_{L^{\gamma(a)}\left(0 . T ; L^{a}\right)} \\
\cdot\left\|\left(J \psi_{1}, J \psi_{2}\right)\right\|_{L^{\gamma(\rho)}\left(0, T ; L^{\rho}\right)}
\end{aligned}
$$

where $\|(u, v)\|_{X}:=\|u\|_{X}+\|v\|_{X}$.

Proof. Noticing that

$$
\begin{aligned}
\nabla\left(V w_{j}\right) & =\nabla V w_{j}+V \nabla w_{j}, \\
\nabla V & =C \nabla\left(\frac{1}{r} *\left(\left|w_{1}\right|^{2}-\left|w_{2}\right|^{2}\right)\right)=\frac{C}{r} * \nabla\left(\left|w_{1}\right|^{2}-\left|w_{2}\right|^{2}\right) \\
& =\frac{C}{r} *\left(w_{1} \nabla \bar{w}_{1}+\nabla w_{1} \bar{w}_{1}-w_{2} \nabla \bar{w}_{2}-\nabla w_{2} \bar{w}_{2}\right),
\end{aligned}
$$

we have for $\frac{1}{\rho^{\prime}}=\frac{1}{\rho}+\frac{2}{a}+\frac{1}{3}-1$

$$
\left\|\nabla\left(V w_{j}\right)\right\|_{L^{\rho^{\prime}}} \leqslant C\left(\left\|w_{1}\right\|_{L^{a}}^{2}+\left\|w_{2}\right\|_{L^{a}}^{2}\right)\left(\left\|\nabla w_{1}\right\|_{L^{\rho}}+\left\|\nabla w_{2}\right\|_{L^{\rho}}\right) .
$$

Thus, we can get

$$
\begin{aligned}
\left\|J\left(V \psi_{j}\right)\right\|_{L^{\rho^{\prime}}} & =\varepsilon|t|\left\|\nabla\left(V w_{j}\right)\right\|_{L^{\rho^{\prime}}} \leqslant C \varepsilon|t|\left(\left\|w_{1}\right\|_{L^{a}}^{2}+\left\|w_{2}\right\|_{L^{a}}^{2}\right)\left(\left\|\nabla w_{1}\right\|_{L^{\rho}}+\left\|\nabla w_{2}\right\|_{L^{\rho}}\right) \\
& \leqslant C\left(\left\|\psi_{1}\right\|_{L^{a}}^{2}+\left\|\psi_{2}\right\|_{L^{a}}^{2}\right)\left(\left\|J \psi_{1}\right\|_{L^{\rho}}+\left\|J \psi_{2}\right\|_{L^{\rho}}\right),
\end{aligned}
$$


which implies the desired result.

Lemma 3.5 (Estimate V). We have the following estimate

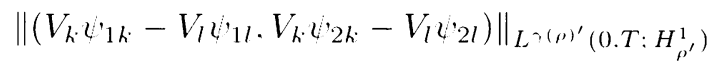

$$
\begin{aligned}
& \leqslant C T^{1 / 2}\left\|\left(\psi_{1 k}, \psi_{2 k}, \psi_{1 l}, \psi_{2 l}\right)\right\|_{L,(a)}^{2}\left(0 . T: H_{l \prime}^{1}\right) \\
& \text { - }\left\|\left(\psi_{1 k}-\psi_{1 l}, \psi_{2 k}-\psi_{2 l}\right)\right\|_{L^{\gamma(p)}\left(0 . T: H_{\rho}^{1}\right)} .
\end{aligned}
$$

Proof. Since

$$
\begin{aligned}
& C\left(V\left(\psi_{1 k}, \psi_{2 k}\right) \psi_{j k}-V\left(\psi_{1 l}, \psi_{2 l}\right) \psi_{j l}\right) \\
= & \left(\frac{1}{r} *\left(\left|\psi_{1 k}\right|^{2}-\left|\psi_{2 k}\right|^{2}\right)\right) \psi_{j k}-\left(\frac{1}{r} *\left(\left|\psi_{1 l}\right|^{2}-\left|\psi_{2 l}\right|^{2}\right)\right) \psi_{j l} \\
= & \left(\frac{1}{r} *\left(\left|\psi_{1 k}\right|^{2}-\left|\psi_{2 k}\right|^{2}\right)\right)\left(\psi_{j k}-\psi_{j l}\right)+\psi_{j l}\left[\frac{1}{r} *\left(\left(\left|\psi_{1 k}\right|^{2}-\left|\psi_{1 l}\right|^{2}\right)-\left(\left|\psi_{2 k}\right|^{2}-\left|\psi_{2 l}\right|^{2}\right)\right)\right] \\
= & \left(\frac{1}{r} *\left(\left|\psi_{1 k}\right|^{2}-\left|\psi_{2 k}\right|^{2}\right)\right)\left(\psi_{j k}-\psi_{j l}\right)+\left[\frac{1}{r} *\left(\left(\bar{\psi}_{1 k}-\bar{\psi}_{1 l}\right) \psi_{1 k}\right)+\frac{1}{r} *\left(\left(\psi_{1 k}-\psi_{1 l}\right) \bar{\psi}_{1 l}\right)\right. \\
& \left.+\frac{1}{r} *\left(\left(\bar{\psi}_{2 k}-\bar{\psi}_{2 l}\right) \psi_{2 k}\right)+\frac{1}{r} *\left(\left(\psi_{2 k}-\psi_{2 l}\right) \bar{\psi}_{2 l}\right)\right] \psi_{j l},
\end{aligned}
$$

we have the desired result by the Hölder inequality:

4. The proof of the existence. In this section. we will prove the local existence of the Cauchy problem (BDNLSP) with the initial data (1.3) first. Let $S(t):=e^{\frac{1}{2} i \varepsilon \Delta t}$ and consider the integral equation

$$
\psi_{j}(t)=S(t) \varphi_{j}-\frac{1}{\varepsilon} i \int_{0}^{t} S(t-\tau)\left(q_{j} V(\tau)+h_{j}\left(\left|\psi_{j}(\tau)\right|^{2}\right)\right) \psi_{j}(\tau) \mathrm{d} \tau
$$

Define the workspace $(\mathcal{D}, d)$ as

$$
\mathcal{D}:=\left\{\left(\psi_{j}\right)_{j=1.2}:\left\|\psi_{j}\right\|_{L^{x}\left(0 . T: H^{1}\right) \cap L^{-(a)}\left(0 . T: H_{a l}^{1}\right)} \leqslant M \text {. for any } a \in[2,6)\right\} .
$$

with the distance

$$
d\left(\left(\psi_{1 k}, \psi_{2 k}\right),\left(\psi_{1 l}, \psi_{2 l}\right)\right)=\left\|\left(\psi_{1 k}-\psi_{1 l}, \psi_{2 k}-\psi_{2 l}\right)\right\|_{L \gamma(\rho)\left(0 . T ; H_{\rho}^{1}\right)}
$$

where $M \geqslant 2 \max _{j=1.2}\left\|\varphi_{j}\right\|_{H^{1}}$ and $\rho \in[2,6)$. It is clear that $(\mathcal{D}, d)$ is a Banach space. Let us consider the mapping $\mathcal{T}=\mathcal{T}_{1} \otimes \mathcal{T}_{2}:(\mathcal{D}, d) \rightarrow(\mathcal{D}, d)$ defined by

$$
\mathcal{T}_{j}: \psi_{j}(t) \mapsto S(t) \varphi_{j}-\frac{1}{\Xi} i \int_{0}^{t} S(t-\tau)\left(q_{j} V(\tau)+h_{j}\left(\left|\psi_{j}(\tau)\right|^{2}\right)\right) \psi_{j}(\tau) \mathrm{d} \tau, j=1.2 .
$$


By Lemmas 3.1-3.5 and the Strichartz estimates (cf. [5]), we have

$$
\begin{aligned}
\left\|\mathcal{T}_{j} \psi_{j}\right\|_{L^{\gamma(\rho)}\left(0 . T: H_{\rho}^{1}\right)} \leqslant & \left\|\varphi_{j}\right\|_{H^{1}}+\left\|V \psi_{j}\right\|_{L^{\gamma(\rho)^{\prime}}\left(0 . T: H_{\rho^{\prime}}^{1}\right)}+\left\|h_{j}\left(\left|\psi_{j}\right|^{2}\right) \psi_{j}\right\|_{L^{\gamma(\rho)^{\prime}}\left(0 . T: H_{\rho^{\prime}}^{1}\right)} \\
\leqslant & \left\|\varphi_{j}\right\|_{H^{1}}+C T^{1 / 2}\left(\sum_{j=1}^{2}\left\|\psi_{j}\right\|_{L^{\gamma(a)}\left(0 . T: H_{a}^{1}\right)}^{2}\right)\left\|\psi_{j}\right\|_{L^{\gamma(\rho)}\left(0 . T: H_{\rho}^{1}\right)} \\
& \quad+C T^{1-2 / \gamma(\rho)}\left\|\psi_{j}\right\|_{L^{\infty}\left(0, T: H^{1}\right)}^{2 \gamma_{j}}\left\|\psi_{j}\right\|_{L^{\gamma(\rho)}\left(0 . T: H_{\rho}^{1}\right)} \\
& \leqslant M / 2+\left(C T^{1 / 2} M^{2}+C T^{1-2 / \gamma(\rho)} M I^{2 \gamma^{\prime} j}\right) M I \\
\leqslant & M I .
\end{aligned}
$$

where we have taken $\mathrm{T}$ so small that $C T^{1 / 2} M^{2}+C T^{1-2 / \gamma(\rho)} M I^{2 \gamma_{j}}<\frac{1}{2}$. Similar to the above, a straightforward computation shows that it holds

$$
\begin{aligned}
& \left\|\mathcal{T}\left(\psi_{1 k}, \psi_{2 k}\right)-\mathcal{T}\left(\psi_{1 l}, \psi_{2 l}\right)\right\|_{L^{\gamma(\rho)}\left(0, T: H_{\rho}^{1}\right)} \\
\leqslant & \frac{1}{2}\left\|\left(\psi_{1 k}-\psi_{1 l}, \psi_{2 k}-\psi_{2 l}\right)\right\|_{L^{\gamma(\rho)}\left(0 . T: H_{\rho}^{1}\right)} .
\end{aligned}
$$

Hence, $\mathcal{T}$ is a contracted mapping from the Banach space $(\mathcal{D}, d)$ to itself. By the Banach contraction mapping principle, we know that there exists a unique solution $\left(\psi_{1} . \psi_{2}\right) \in$ $L^{\gamma(\rho)}\left(0, T ; H_{\rho}^{1}\right) \times L^{\gamma(\rho)}\left(0, T ; H_{\rho}^{1}\right)$ to the (BDNLSP) system with the initial data (1.3). From (4.1), (3.4) and (3.5), we may easily obtain $J \psi_{1}, J \psi_{2} \in L^{\gamma(\rho)}\left(0 . T ; L^{\rho}\right)$ with the help of Lemmas 3.3-3.4. Thus, we can use the standard argument (cf. [3]) to extend it to a global one satisfying for any $T>0$

$$
\psi_{1}(t, x), \psi_{2}(t, x) \in \mathcal{C}\left(\mathbb{R} ; \Sigma\left(\mathbb{R}^{3}\right)\right) \cap L^{\infty}\left(\mathbb{R} ; H^{1}\left(\mathbb{R}^{3}\right)\right) \cap L^{\gamma(\rho)}\left(-T, T ; H_{\rho}^{1}\left(\mathbb{R}^{3}\right)\right)
$$

and prove the uniqueness of the global solution. We omit the details.

5. Large time behavior of the solution. By the pseudo-conformal conservation law, we get for $d=3$

$$
\lambda^{2} t^{2}\|\nabla V\|_{2}^{2} \leqslant I+\lambda^{2} \int_{1}^{t} \tau\|\nabla V(\tau)\|_{2}^{2} \mathrm{~d} \tau
$$

where $I:=\sum_{j=1}^{2}\left\||x| \psi_{j}\right\|_{2}^{2}+\lambda^{2} \int_{0}^{1} \tau\|\nabla V(\tau)\|_{2}^{2} \mathrm{~d} \tau$.

From the Gronwall inequality, we have

$$
\|\nabla V\|_{2} \leqslant \frac{I^{1 / 2}}{\lambda}|t|^{-1 / 2}
$$


By the energy conservation law and the Sobolev embedding theorem, we obtain

$$
\begin{aligned}
\lambda^{2}\|\nabla V\|_{2}^{2} & \leqslant \varepsilon^{2} \sum_{j=1}^{2}\left\|\nabla \varphi_{j}\right\|_{2}^{2}+\|\nabla V(0)\|_{2}^{2}+\sum_{j=1}^{2} \frac{2 a_{j}^{2}}{\gamma_{j}+1}\left\|\varphi_{j}\right\|_{2\left(\gamma_{j}+1\right)}^{2\left(\gamma_{j}+1\right)} \\
& \leqslant C\left(\varepsilon \cdot \gamma_{j} \cdot\left\|\varphi_{j}\right\|_{H^{1}}\right)+\|\nabla V(0)\|_{2}^{2} . \\
\|\nabla V(0)\|_{2} & =C\left\|\nabla\left(\frac{1}{|x|} *\left(\left|\varphi_{1}\right|^{2}-\left|\varphi_{2}\right|^{2}\right)\right)\right\|_{2} \leqslant C\left\|\frac{1}{|x|^{2}} *\left(\left|\varphi_{1}\right|^{2}-\left|\varphi_{2}\right|^{2}\right)\right\|_{2} \\
& \leqslant C \sum_{j=1}^{2}\left\|\varphi_{j}\right\|_{L^{12 / 5}}^{2} \leqslant C \sum_{j=1}^{2}\left\|\varphi_{j}\right\|_{H^{1}}^{2} \\
& \leqslant C\left(\varepsilon, \gamma_{j},\left\|\varphi_{j}\right\|_{H^{1}}\right) .
\end{aligned}
$$

Therefore, we have the estimate

$$
\|\nabla V\|_{2} \leqslant \frac{C}{\lambda}|t|^{-\frac{1}{2}}
$$

By the Sobolev embedding theorem and the pseudo-conformal conservation law, we have

$$
\begin{aligned}
\left\|\psi_{j}\right\|_{\rho}=\left\|\mathcal{M}(-t) \psi_{j}\right\|_{\rho} & \leqslant C\left\|\nabla \mathcal{M}(-t) \psi_{j}\right\|_{2}^{2 / \gamma(\rho)}\left\|\mathcal{M}(t) \psi_{j}\right\|_{2}^{1-\gamma(\rho)} \\
& \leqslant C\left\|\mathcal{M}(-t)\left(\frac{x}{i \varepsilon t} \psi_{j}+\nabla \psi_{j}\right)\right\|_{2}^{2 / \gamma(\rho)}\left\|\psi_{j}\right\|_{2}^{1-\gamma(\rho)} \\
& \leqslant C|t|^{-1 / \gamma(\rho)} .
\end{aligned}
$$

From the above and the Hardy-Littlewood-Sobolev incquality, we obtain

$$
\begin{aligned}
& \|\nabla V(t)\|_{\rho} \leqslant C|t|^{-\left(1-\frac{3}{2 \rho}\right)} . \quad \forall \rho \in\left(\frac{3}{2}, \infty\right), \forall|t| \geqslant 1, \\
& \|V(t)\|_{\rho} \leqslant C|t|^{-\frac{1}{2}\left(1-\frac{3}{\rho}\right)} . \quad \forall \rho \in(3, \infty), \forall|t| \geqslant 1 .
\end{aligned}
$$

\section{REFERENCES}

[1] J. Bergh and J. Löfström, Interpolation spaces, An introduction, Grundlehren der Mathematischen Wissenschaften, No. 223, Springer-Verlag: Berlin-New York, 1976

[2] F. Brezzi and P. A. Markowich, The three-dimensional Wigner-Poisson problem: existence, uniqueness and approximation, Math. Meth. Appl. Sci., 14, 35-62 (1991)

[3] F. Castella, $L^{2}$ solutions to the Schrödinger-Poisson system: existence, uniqueness, time behaviour, and smoothing effects, Math. Models Methods Appl. Sci., 7(8), 1051-1083 (1997)

[4] A. Jüngel and S. Wang, Convergence of nonlinear Schrödinger-Poisson systems to the compressible Euler equations, Comm. Part. Diff. Eqs. 28, 1005-1022 (2003)

[5] M. Keel and T. Tao, Endpoint Strichartz estimates, Amer. J. Math., 120, 955-980 (1998)

[6] H. L. Li and C. K. Lin, Semiclassical limit and well-posedness of nonlinear Schrodinger-Poisson systems, Electron. J. Diff. Eqns., 2003, No. 93, pp. 1-17 (2003)

[7] P. A. Markowich, G. Rein and G. Wolansky. Existence and nonlinear stability of stationary states of the Schrödinger-Poisson system, J. Statist. Phys., 106(5-6), 1221-1239 (2002)

[8] M. E. Taylor, Tools for PDE, Pseudodifferential operators, paradifferential operators, and layer potentials. Mathematical Surveys and Monographs, 81. American Mathematical Society, Providence, RI, 2000

[9] B. X. Wang, Large time behavior of solutions for critical and subcritical complex Ginzburg-Landau equations in $H^{1}$, Science in China (Series A), 46(1), 64-74 (2003) 